\title{
Clinical significance of the presence of anti- human leukocyte antigen-donor specific antibody in kidney transplant recipients with allograft dysfunction
}

Byung Ha Chung ${ }^{1,2}$, Jeong Ho Kim, ${ }^{1,2}$ Bum Soon Choi ${ }^{1,2}$, Cheol Whee Park ${ }^{1,2}$, Ji-Il Kim³ ${ }^{3}$ In Sung Moon ${ }^{3}$, Yong-Soo Kim ${ }^{1,2}$, Yeong Jin $\mathrm{Choi}^{4}$, Eun-Jee $\mathrm{Oh}^{1,5}$, and Chul Woo Yang,

${ }^{1}$ Transplant Research Center, ${ }^{2}$ Division of Nephrology, Department of Internal Medicine, Departments of ${ }^{3}$ Surgery, ${ }^{4}$ Hospital Pathology, and ${ }^{5}$ Laboratory Medicine, College of Medicine, Seoul St. Mary's Hospital, The Catholic University of Korea, Seoul, Korea

Received: March 30, 2016

Revised : May 19, 2016

Accepted: May 28, 2016

\section{Correspondence to}

Chul Woo Yang, M.D.

Department of Internal

Medicine, College of Medicine,

Seoul St. Mary's Hospital, The

Catholic University of Korea, 222

Banpo-daero, Seocho-gu, Seoul

06591, Korea

Tel: +82-2-2258-6037

Fax: +82-2-536-0323

E-mail: yangch@catholic.ac.kr
Background/Aims: This study investigated the clinical significance of detecting anti-human leukocyte antigen-donor specific antibody (HLA-DSA) in kidney transplant recipients (KTRs) requiring indication biopsy owing to allograft dysfunction.

Methods: We analyzed the presence of HLA-DSA in 210 KTRs who took indication biopsy. We divided these cases into two groups, HLA-DSA $(+)(n=52)$ and HLA-DSA $(-)(n=158)$ group, and compared the clinical characteristics, pathological findings, and clinical outcomes of the two groups.

Results: The rates of retransplant, pretransplant sensitization, and HLA-mismatch were significantly higher in HLA-DSA (+) group than in HLA-DSA (-) group ( $p<0.05$ for each comparison). In histologic finding, all types of rejections were more frequent in the former group. Besides, scores of both the T-cell injury markers such as tubulitis, interstitial inflammation, and vasculitis and antibody-mediated injury markers such as peritubular $\mathrm{C}_{4} \mathrm{~d}$ deposition and microvascular inflammation (glomerulitis plus peritubular capillaritis) were higher in HLA-DSA ( + ) group ( $p<0.05$ for each). Notably, allograft outcomes were worse in HLA-DSA (+) group. Further, multivariate analysis showed that presence of HLADSA, advanced interstitial fibrosis/tubular atrophy (interstitial fibrosis plus tubular atrophy $\geq 2$ ), and allograft rejection in biopsy were independent risk factors for allograft failure.

Conclusions: The results of this study showed that presence of HLA-DSA in a case of allograft dysfunction adversely influences allograft outcome, and its detection, irrespective of the result of the allograft biopsy, necessitates intensive monitoring and treatment.

Keywords: Anti-human leukocyte antigen-donor specific antibody; Kidney transplantation; Luminex Single Antigen assay; Allograft biopsy 


\section{INTRODUCTION}

The marked increase over time in detection rate of anti-human leukocyte antigen-donor specific antibody (HLA-DSA) is significantly associated with the decline in allograft function posttransplant and the subsequent allograft failure $[1,2]$. It is well known that HLADSA plays a critical role in the progression of chronic antibody-mediated tissue injury, which in turn, is the most important cause of late allograft failure in kidney transplant recipients (KTRs) $[3,4]$. Therefore, detection and monitoring of HLA-DSA may help in predicting allograft outcomes and planning proper management to prevent decline of allograft function in KTRs.

Most previous studies investigating the role of HLADSA performed serial measurement of HLA-DSA in clinically stable patients without allograft dysfunction and showed that its presence is associated with deterioration of allograft function and subsequent rejection [5-7]. Notably, the clinical significance of HLA-DSA in patients with allograft dysfunction has not been fully investigated. For example, in cases of allograft dysfunction not diagnosed as antibody mediated rejection (AMR) but diagnosed as T cell mediated rejection (TCMR) or calcineurin inhibitor (CNI) toxicity, the clinical significance of detecting HLA-DSA has not yet been fully evaluated.

In this background, we investigated the clinical significance of detecting HLA-DSA in cases with allograft dysfunction. First, we examined the presence of HLA-DSA using Luminex Single Antigen (LSA) assay in all these cases at the time of indication allograft biopsy, which was performed owing to kidney allograft dysfunction. Second, we analyzed the association between the presence of HLA-DSA and specific histological and pathological findings. Finally, we evaluated whether detection of HLA-DSA significantly influences allograft outcome during follow-up.

\section{METHODS}

\section{Baseline characteristics of patients and procedure of indication biopsy}

Between February 2010 and February 2013, a total of 210 KTRs took allograft biopsy due to allograft dysfunction at Seoul St. Mary's Hospital. The definition of allograft dysfunction in this study was a serum creatinine level of $20 \%$ higher than the baseline value or more. Baseline characteristics of patients are given in Table 1 . The procedure of biopsy and the technique of histological diagnosis were based on a previous study [8]. In brief, a 16-gauge biopsy gun was used under ultrasonic localization. Indirect immunofluorescence staining was performed using monoclonal antibodies against complement protein $\mathrm{C}_{4} \mathrm{~d}$ (Biogenesis, Poole, England; dilution, 1:50) for detecting C4d deposition. C4d positivity was defined as diffuse (> $50 \%)$ and linear staining of peritubular capillaries. Microvascular inflammation (MVI) score was calculated by adding glomerulitis (g) and peritubular capillaritis (ptc) scores. Histopathological diagnosis was based on the revised Banff working classification [9-11].

\section{Pretransplant desensitization protocol for pretrans- plant highly sensitized patients}

According to our center's pretransplant desensitization protocol, the target HLA-DSA value at the time of kidney transplant (KT) was a weak or negative level (median fluorescence intensity $[\mathrm{MFI}]<5,000$ ) by LSA assay [12]. In patients with moderate to strong HLA-DSA values (MFI > 5,000), rituximab at a dose of $375 \mathrm{mg} / \mathrm{m} 2$ (MabThera, Genentech Inc., San Francisco, CA, USA) was administered 2 to 3 weeks before transplantation, and plasmapheresis/intravenous immunoglobulin (PP/ IVIG) therapy was initiated 13 days prior to transplantation and administered every other day. In addition, we initiated immune suppressant (IS) treatment 7 days prior to transplantation in these patients. HLA-DSA and crossmatch (XM) testing was performed 2 days prior to the transplant. When HLA-DSA decreased to negative or weak levels and XM testing showed negative conversion, KT was performed. If HLA-DSA results were moderate to strong, or if the XM was positive, we performed additional PP/IVIG three times and subsequently retested the patient for HLA-DSA and XM.

\section{Detection of HLA-DSA and HLA typing}

We examined the presence of HLA-DSA using LSA assay in all cases at the time of allograft biopsy. As described in previous studies, LSA assay for HLA-DSA was performed as per the manufacturer's instructions using Lifecodes LifeScreen Deluxe kits (Tepnel Lifecodes Corp., Stamford, CT, USA) [12,13]. In brief, microbeads 
Table 1. Comparison of clinical characteristics between HLA-DSA (+) and HLA-DSA (-) group

\begin{tabular}{|c|c|c|c|}
\hline Characteristic & HLA-DSA $(+)(n=52)$ & HLA-DSA $(-)(n=158)$ & $p$ value \\
\hline Age at biopsy, yr & $43.7 \pm 10.2$ & $44.0 \pm 11.2$ & 0.87 \\
\hline Male sex & $27(51.9)$ & $99(62.7)$ & 0.11 \\
\hline \multicolumn{4}{|l|}{ Primary renal disease } \\
\hline Chronic glomerulonephritis & $25(48.1)$ & $52(32.9)$ & \\
\hline Diabetes mellitus & $6(11.5)$ & $13(8.2)$ & \\
\hline Hypertension & $3(5.8)$ & $17(10.8)$ & 0.07 \\
\hline ADPKD & $3(5.8)$ & $3(1.9)$ & \\
\hline Others & $15(28.8)$ & $73(46.2)$ & \\
\hline Posttransplant month & $22.4 \pm 41.0$ & $45.7 \pm 65.7$ & $<0.05$ \\
\hline ABO IKT & $7(13.5)$ & $17(10.8)$ & 0.62 \\
\hline \multicolumn{4}{|l|}{ Donor type } \\
\hline LRD & $22(42.3)$ & $81(51 \cdot 3)$ & \\
\hline LURD & $7(13.5)$ & $32(20.3)$ & 0.38 \\
\hline DD & $23(44.2)$ & $45(28.5)$ & \\
\hline Induction therapy & & & 0.04 \\
\hline Anti-thymocyte globulin & $17(32.7)$ & $29(18.4)$ & \\
\hline Basiliximab & $35(67.3)$ & $129(81.6)$ & \\
\hline \multicolumn{4}{|l|}{ Main IS } \\
\hline Tacrolimus & $45(86.5)$ & $135(85.4)$ & \\
\hline Cyclosporine & $6(11.5)$ & $19(12.0)$ & 0.85 \\
\hline Azathioprine & o & $2(1.3)$ & 0.85 \\
\hline Rapamune & $1(1.9)$ & $2(1.3)$ & \\
\hline \multicolumn{4}{|l|}{ Desensitization therapy } \\
\hline RTX/PP/IVIG & $17(32.7)$ & $7(4 \cdot 4)$ & $<0.01$ \\
\hline RTX & $\circ$ & $15(9.5)$ & $<0.01$ \\
\hline No desentization & $35(67.3)$ & $136(86.1)$ & \\
\hline Re-transplantation & $13(25.0)$ & $7(4 \cdot 4)$ & $<0.01$ \\
\hline HLA mismatch number & $3.6 \pm 1.3$ & $2.7 \pm 1.8$ & $<0.05$ \\
\hline High PRA (> 50\%) & $17(32.7)$ & $22(13.9)$ & $<0.01$ \\
\hline HLA-DSA (+) & $17(32.1)$ & $15(9.5)$ & $<0.05$ \\
\hline Positive crossmatch test & $9(17.3)$ & $8(5.1)$ & $<0.05$ \\
\hline
\end{tabular}

Values are presented as mean \pm SD or number $(\%)$.

HLA-DSA, anti-human leukocyte antigen-donor specific antibody; ADPKD, autosomal dominant polycystic kidney disease; ABO IKT, ABO incompatible kidney transplantation; LRD, living related donor; LURD, living unrelated donor; DD, deceased donor; IS, immune suppressant; RTX, rituximab; PP, plasmapheresis; IVIG, intravenous immunoglobulin; PRA, panel reactive antibody.

coated with purified HLA class I/class II glycoproteins were incubated with $12.5 \mu \mathrm{L}$ of patient's serum in 96 well plates for 30 minutes. After three washes with a vacuum manifold, the beads were incubated with $50 \mu \mathrm{L}$ of a 1:10 dilution of R-phycoerythrin-conjugated goat anti-hu- man immunoglobulin $\mathrm{G}$ for 30 minutes. After washing, the test samples were analyzed using the Quick-Type User's Manual Research Use Only program, version 2.4 of the LABScanioo flow cytometer (Luminex Corp., Austin, TX, USA); both positive and negative controls 
were included. The positive criterion was a MFI level of $>1,000$.

HLA typing was performed in all patients and donors using the DNA molecular typing method. Reverse sequence-specific oligonucleotide probes and RELI TM SSO HLA-A, B, C, DR, DQ Typing Kit (Dynal Biotech Ltd., Bromborough, England) were used. In case, in a patient, the anti-HLA antibody detected by LSA assay corresponded to the HLA-type of the donor, it was classified as HLA-DSA. The results were presented as MFI and were classified into four levels based on the peak value of the detected HLA-DSA in a sample: strong, > 10,000; moderate, 5,000 to 10,000; weak, 1,000 to 5,000; and negative, $<1,000$.

\section{Assessment of clinical outcomes}

All subjects were divided into two groups, HLA-DSA (+) and HLA-DSA (-), based on the presence of HLA-DSA at the time of indication biopsy. We compared clinical outcomes between the two groups. The primary outcome of this study was the impact of the presence of HLA-DSA at the time of indication biopsy on the allograft outcome. Secondary outcome was the rate of detection of HLADSA, the factors associated with the development of HLA-DSA, and the association between HLA-DSA and the histological findings of the allograft biopsy. Clinical information was collected using retrospective chart review. Allograft function was assessed using the Modified Diet in the Renal Disease formula [14]. This study was approved by the Institutional Review Board of Seoul's Seoul St. Mary's Hospital (KC11RCMIo687).

\section{Statistical analysis}

Statistical analysis was performed using SPSS version 16.0 (SPSS Inc., Chicago, IL, USA). Data were presented as mean $\pm \mathrm{SD}$ or counts and percentages, depending on the type of data. For continuous variables, means were compared using Student $t$ test. For categorized variables, Pearson chi-square test and Fisher exact test were used. Allograft survival was analyzed using Kaplan-Meier method with a log-rank test. It was censored in case of a patient's death with a functioning allograft. Cox regression analysis was used for multivariate analysis to evaluate risk factors for allograft failure. Results were considered statistically significant if the $p$ value was below 0.05 .

\section{RESULTS}

\section{Detection of HLA-DSA and distribution of HLA-DSA subtypes}

HLA-DSA was detected in a total of 52 cases $(24.8 \%)$ out of 210 cases. HLA-DSA class I was detected in 17 cases (8.1\%); HLA-DSA class II was found in 41 cases (19.5\%); and six cases $(2.9 \%)$ showed both class I and II HLADSA. The most common HLA-DSA subtype was HLADSA-DR (26 cases, 12.4\%) followed by HLA-DSA-DQ (19 cases, 9.0\%) and HLA-DSA-B (12 cases, 5.7\%). Weak HLA-DSA was detected in 35 cases (16.7\%), five cases (2.4\%) showed moderate HLA-DSA, and 12 cases (5.7\%) showed strong HLA-DSA. In comparison between the two groups, HLA-DSA $(+)(\mathrm{n}=52)$ and HLA-DSA $(-)(\mathrm{n}=$ 158), no significant difference in clinical characteristics such as age at biopsy, sex, primary renal disease, donor type, type of main IS, and ABO incompatibility to donor was detected. The posttransplant duration was significantly longer in HLA-DSA (-) group compared to HLADSA $(+)$ group $(45.7 \pm 65.7$ vs. $22.4 \pm 41.0, p<0.05$ ) (Table 1). Regarding pretransplant immunological characteristics, significant differences were found between the two groups. The HLA-mismatch number, incidence of retransplantation, number of patients with high panel reactive antibody, and HLA-DSA at baseline were significantly higher in HLA-DSA (+) group compared to HLA-DSA (-) group (Table 1).

\section{Comparing allograft function and pathological find- ings of HLA-DSA (+) and HLA-DSA (-) groups}

At the time of allograft biopsy, there was no significant difference in allograft function between the two groups (HLA-DSA [+] group, $29.3 \pm 14.5 \mathrm{~mL} / \mathrm{min} / 1.73 \mathrm{~m}^{2}$ vs. HLADSA [-] group, $\left.31.6 \pm 15.4 \mathrm{~mL} / \mathrm{min} / 1.73 \mathrm{~m}^{2}, p>0.05\right)$. In contrast, pathological diagnoses/findings based on Banff classification differed significantly between the two groups [9-11]. The incidence rates of not only total rejection (HLA-DSA [+], 80.8\%, 42/52 vs. HLA-DSA [-], 30.4\%, 48/158) (Fig. 1A), but also AMR (HLA-DSA [+], 28.8\%, 15/52 vs. HLA-DSA [-], ०\%, ०/158) and TCMR (HLA-DSA [+], 48.1\%, 25/52 vs. HLA-DSA [-], 30.4\%, 48/158) were significantly higher in HLA-DSA (+) group compared to HLADSA (-) group ( $p<0.05$ for each comparison). In contrast, the incidence of CNI toxicity was significantly higher in the HLA-DSA (-) group $(24.7 \%, 39 / 158)$ than in the HLA- 


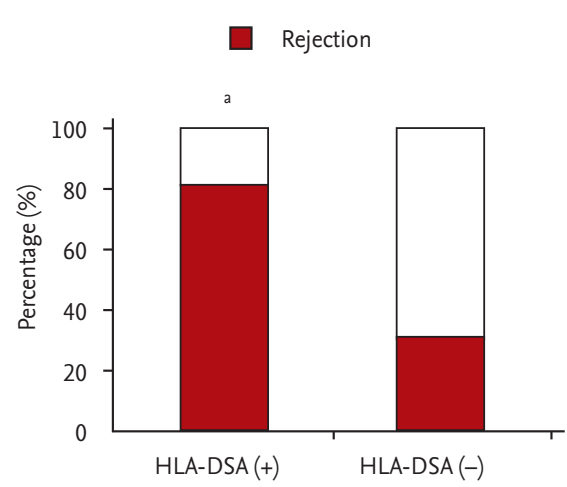

A

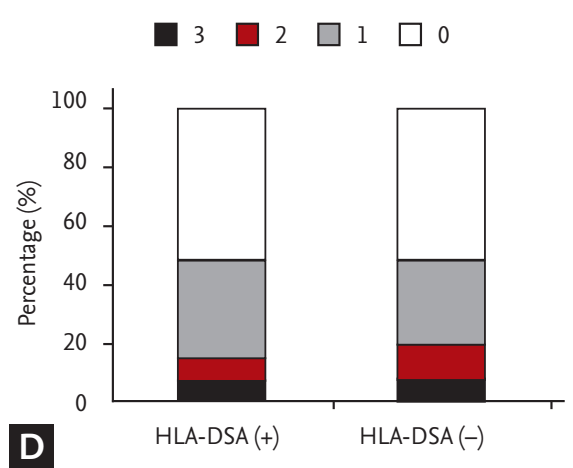

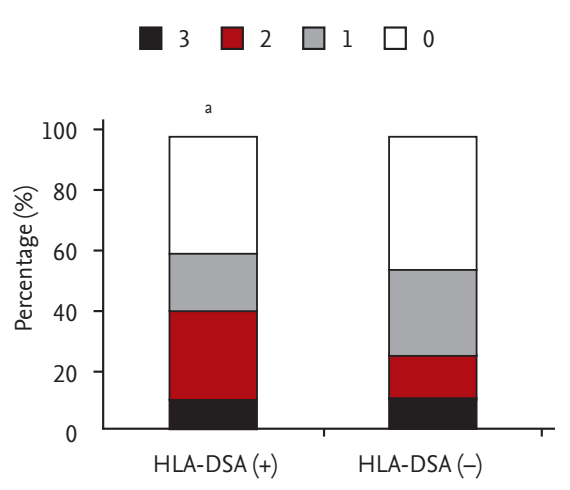

B

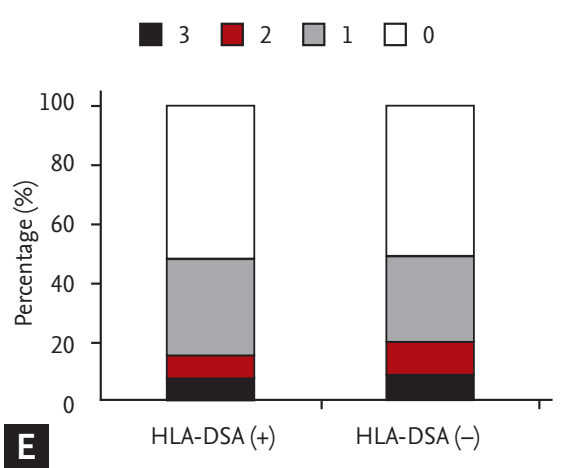

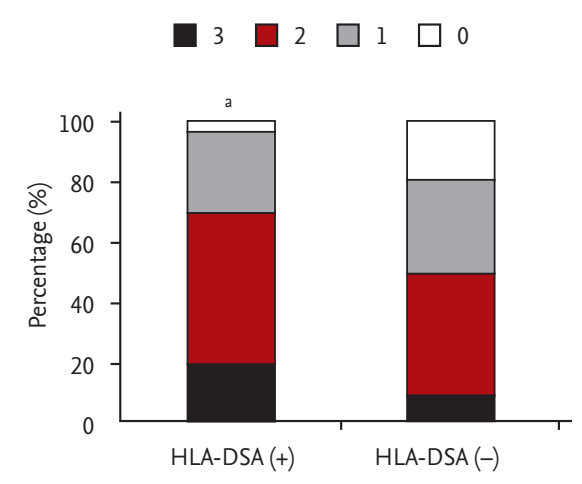

C

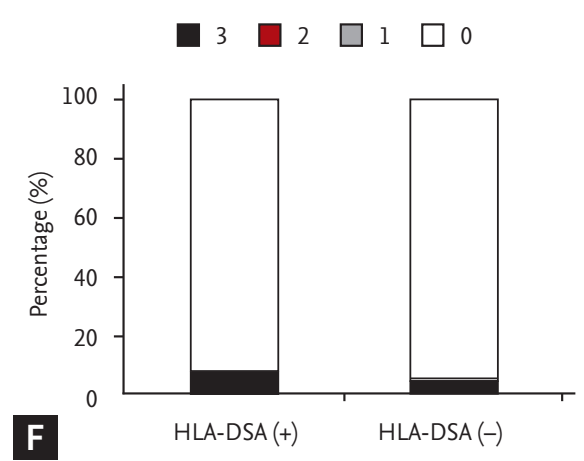

Figure 1. Comparing allograft biopsy finding and the distribution of Banff score in the anti-human leukocyte antigen-donor specific antibody (HLA-DSA) (+) and HLA-DSA (-) groups. (A) Incidence of total allograft rejection was significantly higher in HLA-DSA (+) group compared to HLA-DSA (-) group. Scores of (B) tubulitis and (C) interstitial inflammation were higher in the HLA-DSA (+) group than in the HLA-DSA (-) group. No significant difference was detected in the distribution of (D) interstitial fibrosis, (E) tubular atrophy, and (F) glomerulosclerosis scores between the HLA-DSA (+) and HLA-DSA (-) groups. ${ }^{a} p<0.05$ vs. HLA-DSA (-) group.

DSA (+) group (9.6\%, 5/52, p< 0.05) (Table 2). When we compared the biopsy findings of KTRs who progressed to allograft failure between HLA-DSA (+) and HLA-DSA $(-)$ group, AMR showed increasing tendency as a cause for allograft failure in HLA-DSA (+) group, but it did not reach statistical significance (Table 3).

\section{Comparing distribution of Banff and microvascular inflammation scores}

Regarding distribution of Banff score, $t$ (tubulitis), $i$ (interstitial inflammation) scores were higher in the HLADSA (+) group than in the HLA-DSA (-) group $(p<0.05)$ (Fig. ${ }_{1 B}$ and ${ }_{1 C}$ ). In contrast, ci (interstitial fibrosis), ct (tubular atrophy) and cg (glomerular sclerosis) scores did not differ significantly between the two groups ( $p>$ 0.05 ) (Fig. 1D-1F). Higher scores of histological markers associated with activation of humoral immunity such as peritubular $\mathrm{C}_{4} \mathrm{~d}$ deposition, g, v (vasculitis), and ptc scores were distributed in HLA-DSA (+) group compared to HLA-DSA (-) group ( $p<0.05$ ) (Fig. 2A-2D). Lastly, higher MVI score, calculated by adding $g$ and ptc scores, was distributed in HLA-DSA (+) group $(p<0.05)$ (Fig. $2 \mathrm{E})$.

\section{Comparing allograft outcomes between HLA-DSA $(+)$ and HLA-DSA $(-)$ groups}

Fig. 3 compares clinical outcomes between the HLADSA (+) and HLA-DSA (-) groups. The incidence of recurrent or newly developed acute rejection within 6 months $(19.2 \%$ [10/52] vs. $6.3 \%$ [10/158]) and that of steroid-resistant rejection (32.7\% [17/52] vs. $7.6 \%$ [12/158]) were significantly higher in HLA-DSA (+) group compared to HLA-DSA (-) group ( $p<0.05$ for each) (Fig. $3 A$ and $\left.{ }_{3} \mathrm{~B}\right)$. When only rejection cases were included, incidence of recurrent or newly developed rejection with- 
Table 2. Comparison of histologic diagnosis between HLA-DSA (+) group and HLA-DSA (-) group

\begin{tabular}{|c|c|c|c|}
\hline Variable & $\operatorname{HLA}-\mathrm{DSA}(+)(\mathrm{n}=52)$ & HLA-DSA $(-)(n=158)$ & $p$ value \\
\hline TCMR & $25(48)$ & $48(30)$ & $<0.001$ \\
\hline Acute or chronic AMR & $17(33)$ & 0 & $<0.001$ \\
\hline CNI toxicity & $5(10)$ & $39(25)$ & $<0.001$ \\
\hline Recurrent GN & o & $18(11)$ & $<0.001$ \\
\hline Borderline change & $2(4)$ & $18(11)$ & $<0.001$ \\
\hline Acute tubular necrosis & o & $8(5)$ & $<0.001$ \\
\hline BKVAN & $1(2)$ & $6(4)$ & $<0.001$ \\
\hline Normal and others & $4(8)$ & $29(18)$ & $<0.001$ \\
\hline
\end{tabular}

Values are presented as number (\%).

HLA-DSA, anti-human leukocyte antigen-donor specific antibody; TCMR, T cell mediated rejection; AMR, antibody mediated rejection; CNI, calcineurin inhibitor; GN, glomerulonephritis; BKVAN, BK virus associated nephropathy.

Table 3. Comparison of histologic diagnosis in patients with allograft failure between HLA-DSA (+) group and HLA-DSA (-) group

\begin{tabular}{lccc}
\hline Variable & HLA-DSA $(+)(\mathrm{n}=17)$ & HLA-DSA $(-)(\mathrm{n}=26)$ & $p$ value \\
\hline TCMR & $11(65)$ & $15(58)$ & $<0.001$ \\
Acute or chronic AMR & $4(24)$ & 0 & $<0.001$ \\
CNI toxicity & $1(6)$ & $5(19)$ & $<0.001$ \\
BKVAN & $1(2)$ & $6(4)$ & $<0.001$ \\
Recurrent GN & 0 & $4(15)$ & $<0.001$ \\
\hline
\end{tabular}

Values are presented as number (\%).

HLA-DSA, anti-human leukocyte antigen-donor specific antibody; TCMR, T cell mediated rejection; AMR, antibody mediated rejection; CNI, calcineurin inhibitor; BKVAN, BK virus associated nephropathy; GN, glomerulonephritis.

in 6 months $(21.4 \%[9 / 42]$ vs. $12.5 \%$ [6/48]) showed an increasing tendency ( $p=0.08$ ) (Fig. $3 \mathrm{C}$ ), whereas incidence of steroid-resistant rejection (40.5\% [17/42] vs. $25 \%$ [12/48]) showed a significant increase $(p<0.05)$ (Fig. 3D). Therefore, allograft survival rate was lower in HLA-DSA (+) group than in HLA-DSA (-) group not only in the entire cohort, but also in the rejection and non-rejection groups ( $p<0.05$ for each) (Fig. $3 \mathrm{E}-3 \mathrm{G}$ ). Multivariate analysis showed that presence of HLA-DSA, especially class I, advanced Interstitial fibrosis/tubular atrophy (IF/TA; ci $+c t \geq 2$ ), and allograft rejection in allograft tissue were independent risk factors for allograft failure (Table 4).

\section{DISCUSSION}

Application of Luminex technology in measuring HLA-
DSA enables accurate determination of humoral immunity in KTRs $[6,15,16]$. In our previous study, we reported the clinical usefulness of pretransplant HLA-DSA measured by Luminex technique in predicting posttransplant outcome $[12,17]$. In this study, we investigated the clinical usefulness of measuring posttransplant HLA-DSA, especially in cases of allograft dysfunction, and found that it is associated with higher incidence of rejection and more active pathological findings despite similar allograft function. We also found it to be an independent risk factor for allograft failure.

To start with, we studied the distribution of HLA-DSA according to class and strength and found that the pattern of posttransplant HLA-DSA was different from that of pretransplant HLA-DSA [12,17]. Before transplant, the frequencies of class I and class II HLA-DSA were the same; however, during the posttransplant period, 

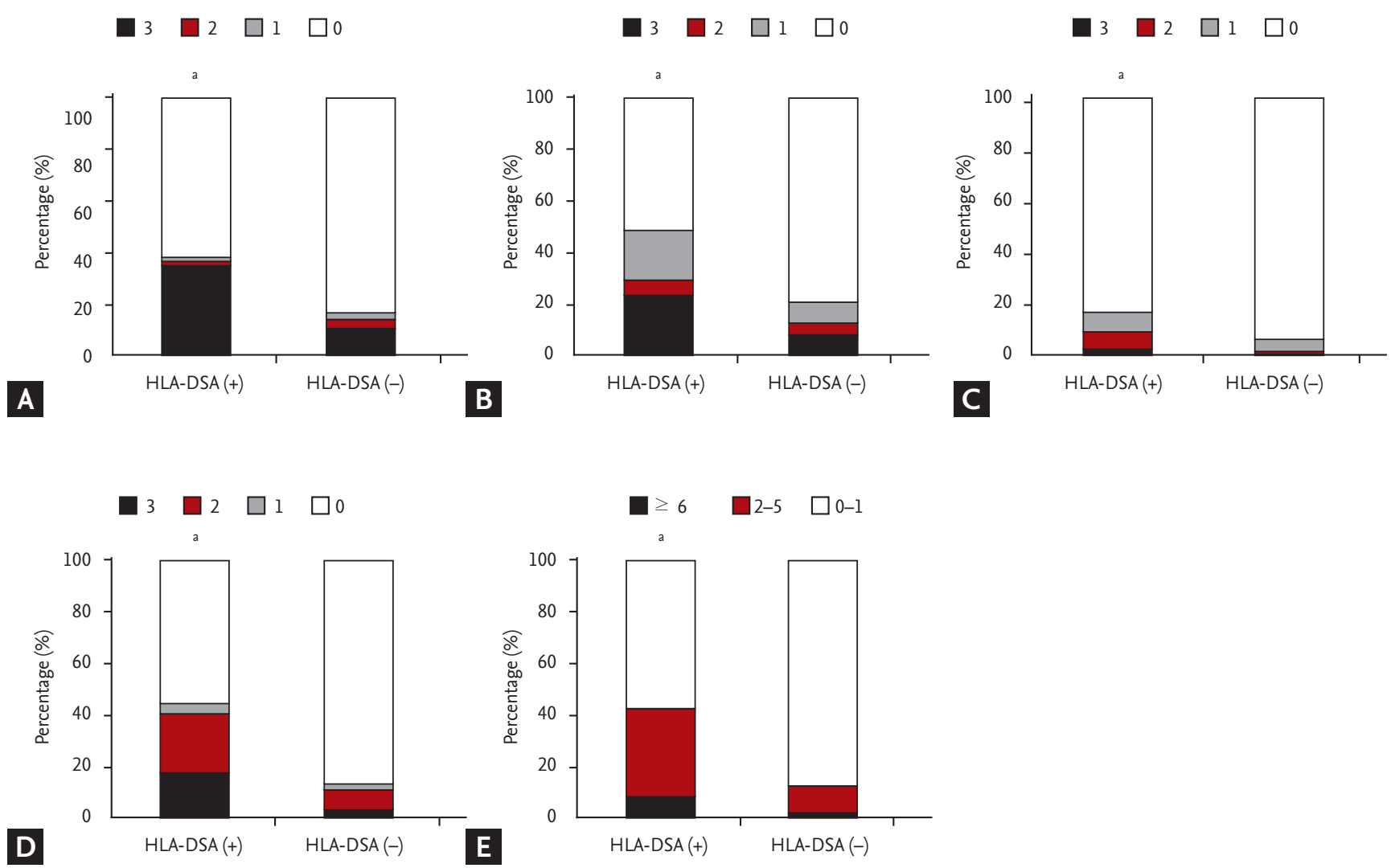

Figure 2. Comparing histological markers associated with humoral immunity between the donor specific anti-human leukocyte antigen antibody (HLA-DSA) (+) and HLA-DSA (-) groups. (A) C4d, (B) glomerulitis, (C) vasculitis, and (D) peritubular capillaritis scores were higher in the HLA-DSA (+) group than in the HLA-DSA (-) group. (E) In addition, the proportion of cases with significant microvascular inflammation score $(\geq 2)$ was higher in the HLA-DSA $(+)$ group. ${ }^{a} p<0.05$ vs. HLA-DSA $(-)$ group.

HLA-DSA class II, especially HLA-DSA-DR and HLADSA-DQ, were more frequently detected. This finding is consistent with results of previous studies that reported up-regulation of class II HLA-DSA de novo after transplantation $[7,18]$. Regarding strength of HLA-DSA, most posttransplant cases, like the pretransplant ones, showed weak HLA-DSA [12,17].

Next, we divided cases into two groups, HLA-DSA (+) and HLA-DSA (-), based on the presence of HLA-DSA detected at the time of allograft biopsy. We compared the clinical and immunological characteristics between the two groups and found that the incidence of pretransplant sensitization was significantly higher in HLA-DSA (+) group. This is consistent with the findings of the previous studies that showed the role of pretransplant sensitization in de novo appearance of posttransplant HLADSA [19,20]. Interestingly, in our study, posttransplant duration was longer in HLA-DSA (-) group in contrast with the results of the previous studies which showed gradual increase in de novo detection of HLA-DSA with time after KT $[5,7,21]$. This may be due to the fact that we studied cases with allograft dysfunction, whereas previous studies mainly included clinically stable patients. In patients without HLA-DSA, the main cause of allograft dysfunction is not allograft rejection but other reasons such as CNI toxicity, as also shown in our study, and compared to allograft rejection, allograft dysfunction requires longer time to develop [22].

Thereafter, we compared pathological findings of the allograft tissue. As expected, the incidence of allograft rejection was significantly higher in HLA-DSA (+) group. Interestingly, not only the incidence of AMR, but also that of TCMR was significantly higher in HLA-DSA (+) group compared to HLA-DSA (-) group. It is well known that complex interactions between $\mathrm{T}$ cells and $\mathrm{B}$ cells are involved in the activation of immune system, which 
A

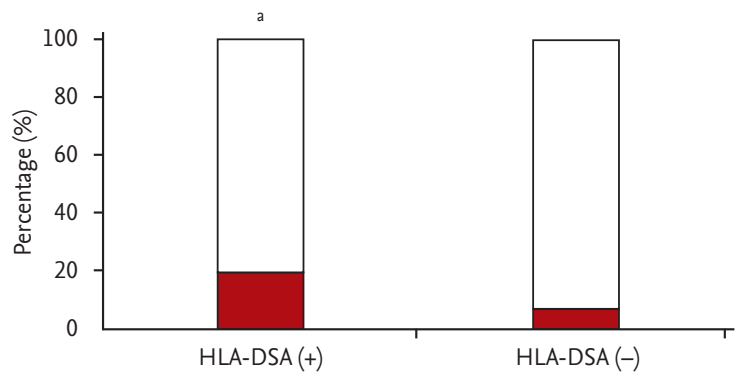

C

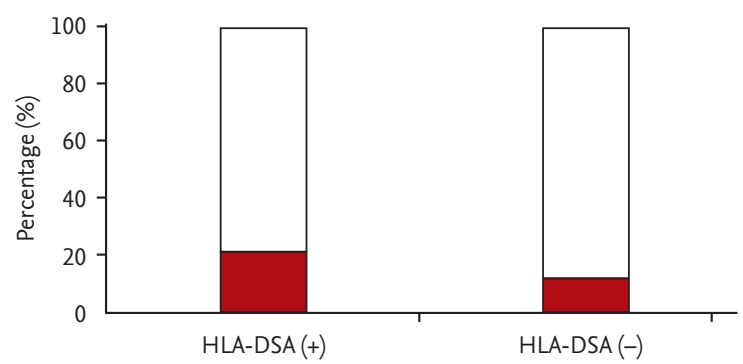

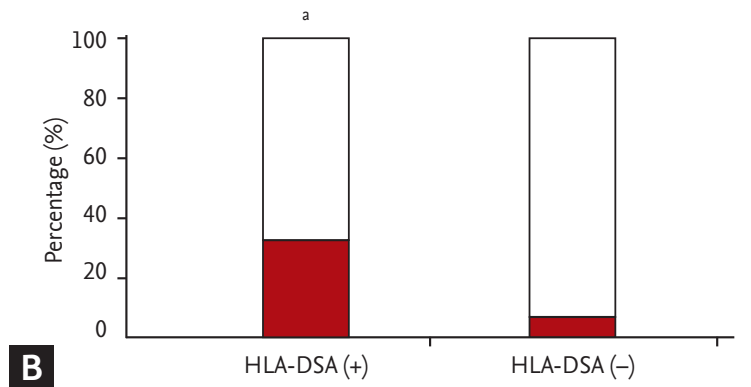

D

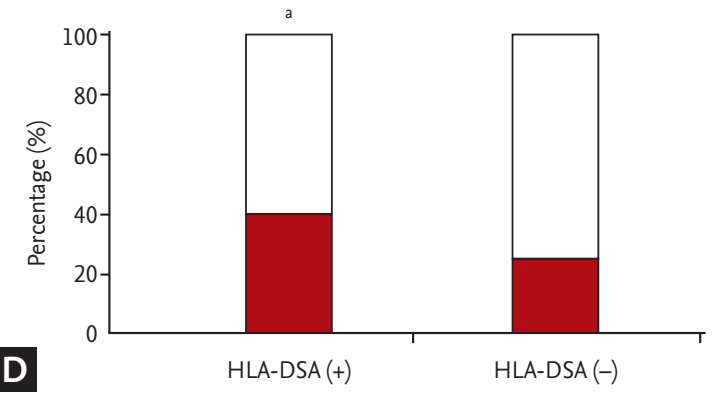

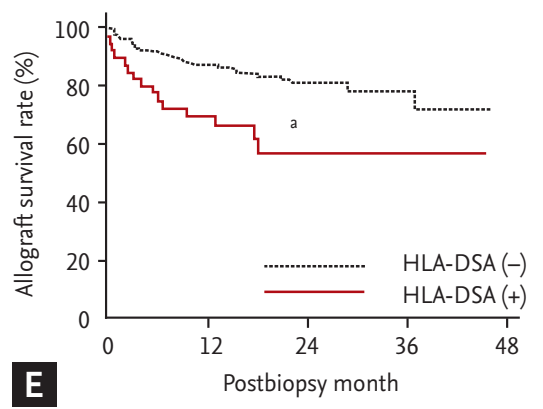
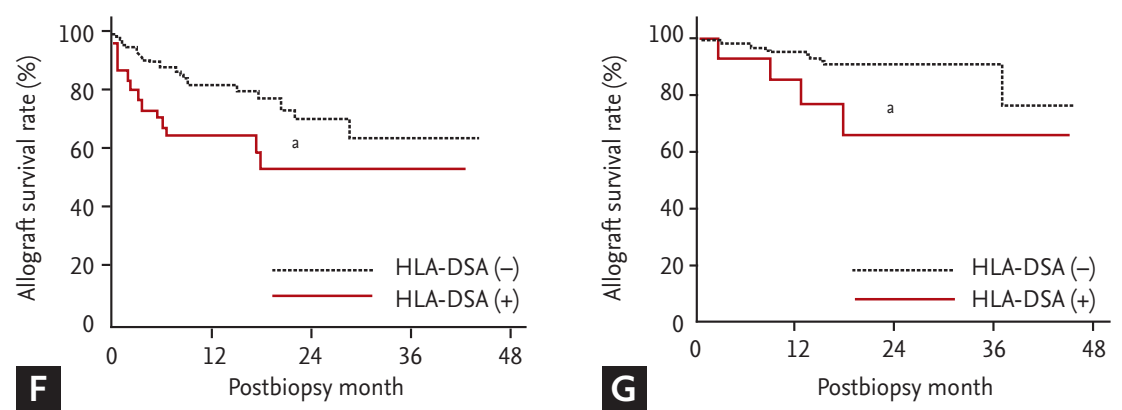

Figure 3. Comparing clinical outcomes after allograft biopsy between the donor specific anti-human leukocyte antigen antibody (HLA-DSA) (+) and HLA-DSA (-) groups. Comparing incidence of (A) repeated rejection within 6 months of allograft biopsy and (B) steroid-resistant rejection between the HLA-DSA (+) and HLA-DSA (-) groups in the entire cohort. Comparing incidence of $(\mathrm{C})$ repeated rejection within 6 months of allograft biopsy and (D) steroid-resistant rejection between the HLADSA (+) and HLA-DSA (-) groups among the rejection cases. Comparing allograft survival rate after biopsy between the HLADSA (+) and HLA-DSA (-) groups (E) in the entire cohort, (F) among the rejection cases, and (G) among the non-rejection cases. ${ }^{a} p<0.05$ vs. HLA-DSA $(-)$ group.

results in allograft rejection; therefore, $\mathrm{T}$ cell activation could be involved in de novo development of HLA-DSA or vice versa [23-27]. Besides, in our study, CNI toxicity was more frequent in the HLA-DSA (-) group. This may be because allograft dysfunction requires longer time to develop posttransplantation resulting in greater exposure to CNI in this group [22].

Regarding pathological findings based on Banff classification, the histological markers associated with activation of local humoral immune system such as C4d score and MVI score, the combination of $g$ and ptc scores, in- creased in the HLA-DSA $(+)$ group, which indicates frequent development of HLA-DSA-induced allograft tissue injury in this group $[28,29]$. In addition, scores of $t$ and $i$ were higher in the HLA-DSA (+) group than in the HLADSA (-) group, which suggests increased rate of TCMR in this group. In previous studies about the role of HLADSA in stable KTRs without allograft dysfunction, the progression of chronic tissue injury mostly presenting as chronic AMR, was the most dominant finding [5-7]. In contrast, ct, ci, and cg scores were similarly distributed in the two groups indicating that advancement 
Table 4. Significant risk factors associated with allograft failure

\begin{tabular}{|c|c|c|c|c|c|c|}
\hline \multirow{2}{*}{ Variable } & \multicolumn{3}{|c|}{ Univariate } & \multicolumn{3}{|c|}{ Multivariate } \\
\hline & HR & $95 \% \mathrm{CI}$ & $p$ value & HR & $95 \% \mathrm{CI}$ & $p$ value \\
\hline Age & 1.007 & $0.980-1.034$ & 0.625 & - & - & - \\
\hline Donor type (DD) & 1.626 & $1.164-2.270$ & 0.004 & 1.327 & $0.938-1.879$ & 0.110 \\
\hline Posttransplant month & 1.005 & $1.000-1.009$ & 0.031 & 1.004 & $0.999-1.009$ & 0.147 \\
\hline Pretransplant sensitization & 0.435 & $0.153-1.240$ & 0.119 & - & - & - \\
\hline Main IS & 1.032 & $0.600-1.775$ & 0.856 & - & - & - \\
\hline Retransplant & 2.442 & $1.312-4.543$ & 0.014 & 1.821 & $0.905-3.662$ & 0.093 \\
\hline HLA-DSA at biopsy & 2.371 & $1.259-4.463$ & 0.007 & 2.847 & $1.461-5.548$ & 0.002 \\
\hline Strong HLA-DSA & 1.970 & $0.700-5.546$ & 0.199 & - & - & - \\
\hline HLA-DSA class I & 4.284 & $2.047-8.967$ & $<0.001$ & 4.786 & $2.134-10.735$ & 0.003 \\
\hline HLA-DSA class II & 1.563 & $0.787-3.102$ & 0.202 & - & - & - \\
\hline Advanced IF/TA & 3.789 & $2.085-6.883$ & 0.000 & 3.438 & $1.689-6.996$ & 0.001 \\
\hline Rejection & 2.834 & $1.538-5.222$ & 0.001 & 2.330 & $1.211-4.483$ & 0.011 \\
\hline
\end{tabular}

HR, hazard ratio; CI, confidence interval; DD, deceased donor; IS, immune suppressant; HLA-DSA, anti-human leukocyte antigen-donor specific antibody; IF/TA, interstitial fibrosis tubular atrophy.

of chronic change is not characteristic findings when HLA-DSA was detected in KTRs with allograft dysfunction in contrast to cases with stable allograft function.

Finally, we investigated the impact of HLA-DSA in cases with allograft dysfunction on future clinical outcomes and found that these outcomes were significantly worse in HLA-DSA (+) group in terms of recurrent rejection, steroid resistance, and lower survival rate of allograft following allograft dysfunction. Interestingly, similar results were found in the sub-analysis involving only the rejection group or only the non-rejection group. Additionally, multivariate analysis showed that presence of HLA-DSA, especially class I antibody, advanced IF/TA, and finding of allograft rejection in biopsy were independent risk factors for allograft failure. These findings suggest that presence of HLA-DSA may be associated with adverse allograft outcome irrespective of histological findings in cases with allograft dysfunction. Previous studies show that HLA-DSA could induce activation of local immune system in allograft tissue resulting in progression of allograft tissue inflammation, corroborated by active pathological findings of our study [30]. Notably, cases with such injuries may be associated with adverse clinical outcomes such as recurrence of rejection, resistance to steroid pulse therapy, and finally allograft failure. Indeed, TCMR or AMR accounts for $88 \%$ of the cause for allograft failure in HLA-DSA (+) group, which is higher than that of HLA-DSA (-) group (57\%) (Table 3).

The limitation of this study is that we did not study the longitudinal change in HLA-DSA before and after the allograft biopsy; hence, we could not assess the actual point of time of development of HLA-DSA and allograft dysfunction. Second, HLA-DSA (+) group already showed higher pre-transplant immunologic risk, which may induce bias during analysis. Lastly, we could not assess the clinically significant value of MFI because the strength of HLA-DSA in our study did not have significant impact on allograft outcome. This is in contrast with the situation before KT, where only the strong HLA-DSA is not clinically relevant [11,12,31]. The reason is unclear, but it may be possible that even a weak HLADSA may develop strong immunogenicity under maintenance immunosuppression.

In conclusion, presence of HLA-DSA in cases with allograft dysfunction is significantly associated with accompanied rejection, active pathological findings, and histological markers for antibody-mediated injury. In addition, presence of HLA-DSA is an independent risk factor for allograft failure. Hence, we suggest that measurement of HLA-DSA should be mandatorily performed in KT'Rs with allograft dysfunction. Moreover, in case HLA-DSA is detected, strict monitoring and intensive 
immunosuppressive therapy may be required even when allograft biopsy does not show allograft rejection.

\section{KEY MESSAGE}

1. Posttransplant detection rate of anti-human leukocyte antigen-donor specific antibody (HLA-DSA) was higher in cases with pretransplant sensitization.

2. Detection of HLA-DSA in cases with allograft dysfunction is associated with active pathological findings, and histological markers for antibody-mediated injury.

3. Presence of HLA-DSA in cases with allograft dysfunction adversely influences allograft outcome irrespective of the result of the allograft biopsy.

\section{Conflict of interest}

No potential conflict of interest relevant to this article was reported.

\section{Acknowledgments}

This study was supported by a grant ( $\left.\mathrm{HI}_{3} \mathrm{C} \mathrm{C}_{23} 32\right)$ of the Korean Health Technology R\&D Project, Ministry of Health and Welfare, Republic of Korea.

\section{REFERENCES}

1. Wiebe C, Gibson IW, Blydt-Hansen TD, et al. Evolution and clinical pathologic correlations of de novo donor-specific HLA antibody post kidney transplant. Am J Transplant 2012;12:1157-1167.

2. Lee PC, Terasaki PI, Takemoto SK, et al. All chronic rejection failures of kidney transplants were preceded by the development of HLA antibodies. Transplantation 2002;74:1192-1194.

3. Sellares J, de Freitas DG, Mengel M, et al. Understanding the causes of kidney transplant failure: the dominant role of antibody-mediated rejection and nonadherence. Am J Transplant 2012;12:388-399.

4. Cosio FG, Gloor JM, Sethi S, Stegall MD. Transplant glomerulopathy. Am J Transplant 2008;8:492-496.

5. Everly MJ, Rebellato LM, Haisch CE, et al. Incidence and impact of de novo donor-specific alloantibody in primary renal allografts. Transplantation 2013;95:410-417.

6. Dieplinger G, Ditt V, Arns W, et al. Impact of de novo donor-specific HLA antibodies detected by Luminex solid-phase assay after transplantation in a group of 88 consecutive living-donor renal transplantations. Transpl Int 2014;27:60-68.

7. Dieplinger G, Everly MJ, Rebellato LM, et al. Changes in successive measures of de novo donor-specific anti-human leukocyte antigen antibodies intensity and the development of allograft dysfunction. Transplantation 2014;98:1097-1104.

8. Chung BH, Lee JY, Kang SH, et al. Comparison of clinical outcome between high and low baseline anti-ABO antibody titers in ABO-incompatible kidney transplantation. Ren Fail 2011;33:150-158.

9. Solez K, Colvin RB, Racusen LC, et al. Banff of classification of renal allograft pathology: updates and future directions. Am J Transplant 2008;8:753-760.

10. Haas M, Sis B, Racusen LC, et al. Banff 2013 meeting report: inclusion of $\mathrm{C}_{4} \mathrm{~d}$-negative antibody-mediated rejection and antibody-associated arterial lesions. Am J Transplant 2014;14:272-283.

11. Cooper JE, Gralla J, Chan L, Wiseman AC. Clinical significance of post kidney transplant de novo DSA in otherwise stable grafts. Clin Transpl 2011:359-364.

12. Chung BH, Choi BS, Oh EJ, et al. Clinical impact of the baseline donor-specific anti-human leukocyte antigen antibody measured by Luminex single antigen assay in living donor kidney transplant recipients after desensitization therapy. Transpl Int 2014;27:49-59.

13. Chung BH, Joo YY, Lee J, et al. Impact of ABO incompatibility on the development of acute antibody-mediated rejection in kidney transplant recipients presensitized to HLA. PLoS One 2015;10:e0123638.

14. Armstrong KA, Campbell SB, Hawley CM, Nicol DL, Johnson DW, Isbel NM. Obesity is associated with worsening cardiovascular risk factor profiles and proteinuria progression in renal transplant recipients. Am J Transplant 2005;5:2710-2718.

15. Vlad G, Ho EK, Vasilescu ER, et al. Relevance of different antibody detection methods for the prediction of antibody-mediated rejection and deceased-donor kidney allograft survival. Hum Immunol 2009;70:589-594.

16. Caro-Oleas JL, Gonzalez-Escribano MF, Gonzalez-Roncero FM, et al. Clinical relevance of HLA donor-specific 
antibodies detected by single antigen assay in kidney transplantation. Nephrol Dial Transplant 2012;27:12311238.

17. Hwang HS, Sun IO, Yoon HE, et al. Antibody monitoring system to support the single-antigen Luminex assay in donor-specific antibody detection. Hum Immunol 2012;73:370-375.

18. Ntokou IS, Iniotaki AG, Kontou EN, et al. Long-term follow up for anti-HLA donor specific antibodies postrenal transplantation: high immunogenicity of HLA class II graft molecules. Transpl Int 2011;24:1084-1093.

19. Gloor JM, Cosio FG, Rea DJ, et al. Histologic findings one year after positive crossmatch or ABO blood group incompatible living donor kidney transplantation. Am J Transplant 2006;6:1841-1847.

20. Ishida H, Furusawa M, Shimizu T, Nozaki T, Tanabe K. Influence of preoperative anti-HLA antibodies on shortand long-term graft survival in recipients with or without rituximab treatment. Transpl Int 2014;27:371-382.

21. Lachmann N, Terasaki PI, Budde K, et al. Anti-human leukocyte antigen and donor-specific antibodies detected by luminex posttransplant serve as biomarkers for chronic rejection of renal allografts. Transplantation 2009;87:15051513.

22. Nankivell BJ, Borrows RJ, Fung CL, O'Connell PJ, Allen $\mathrm{RD}$, Chapman JR. The natural history of chronic allograft nephropathy. N Engl J Med 2003;349:2326-2333.

23. Thomas WR, Hales BJ. T and B cell responses to HDM allergens and antigens. Immunol Res 2007;37:187-199.

24. Ramiscal RR, Vinuesa CG. T-cell subsets in the germinal center. Immunol Rev 2013;252:146-155.

25. Qi H. From SAP-less T cells to helpless B cells and back: dynamic T-B cell interactions underlie germinal center development and function. Immunol Rev 2012;247:24-35.

26. Naemi FM, Carter V, Kirby JA, Ali S. Anti-donor HLA class I antibodies: pathways to endothelial cell activation and cell-mediated allograft rejection. Transplantation 2013;96:258-266.

27. Lobo LJ, Aris RM, Schmitz J, Neuringer IP. Donor-specific antibodies are associated with antibody-mediated rejection, acute cellular rejection, bronchiolitis obliterans syndrome, and cystic fibrosis after lung transplantation. J Heart Lung Transplant 2013;32:70-27.

28. Sis B, Jhangri GS, Riopel J, et al. A new diagnostic algorithm for antibody-mediated microcirculation inflammation in kidney transplants. Am J Transplant 2012;12:11681179.

29. de Kort H, Willicombe M, Brookes P, et al. Microcirculation inflammation associates with outcome in renal transplant patients with de novo donor-specific antibodies. Am J Transplant 2013;13:485-492.

30. Loupy A, Hill GS, Jordan SC. The impact of donor-specific anti-HLA antibodies on late kidney allograft failure. Nat Rev Nephrol 2012;8:348-357.

31. Aubert V, Venetz JP, Pantaleo G, Pascual M. Low levels of human leukocyte antigen donor-specific antibodies detected by solid phase assay before transplantation are frequently clinically irrelevant. Hum Immunol 2009;70:580583. 